\title{
Clinical profile and outcome of cerebral venous sinus thrombosis at tertiary care center
}

\author{
Virendra C. Patil, Kushal Choraria, Neeraj Desai, Sumit Agrawal \\ Department of Medicine, Krishna Institute of Medical Sciences University, Satara, Maharashtra, India
}

\begin{abstract}
Background: Thrombosis of the cerebral venous sinuses (CVST) is an uncommon form of stroke, usually affecting young individuals. Clinical features of CVST are diverse, and for this reason, high degree of clinical suspect is mandatory to diagnose the conditions. Materials and Methods: This study was conducted over a period of 1 year (Jan 2011 to Dec 2011). This was a retrospective, observational, and noninterventional study. This study was conducted in the Department of Medicine at a tertiary care teaching center. Total 50 patients where diagnosis of CVST was confirmed by computed tomography/ magnetic resonance imaging brain venogram were included in this study. All patients with diagnosis of CVST were treated according to the standard protocol and guidelines. Statistical Analysis: The mean and standard deviation were obtained. The Chi-square test was used to analyze the data and $P<0.05$ was considered as statistically significant. Results: Of total 50 patients with diagnosis of CVST, 21 (42\%) were males and $29(58 \%)$ were females with $39 \pm 10$ years and $29 \pm 7$ years, respectively. Total $45(90 \%)$ patients presented with symptoms of headache and vomiting, $13(26 \%)$ had seizures, $12(24 \%)$ had hemiplegia, and $19(38 \%)$ had fever. A total of 13 (26\%) patients had papilledema on fundoscopy. Total 9 ( $31 \%)$ out of 29 patients had diagnosis of CVST during peripartum period. Total $12(24 \%)$ patients had hyperhomocysteinemia. Total $23(46 \%)$ patients had sagittal sinus thrombosis, $10(20 \%)$ had multiple sinus thrombosis, $16(32 \%)$ had sigmoid/transverse sinus thrombosis. There was 1 (2\%) patient who had bilateral cavernous sinus thrombosis, who presented with bilateral proptosis, conjunctival congestion, and external ophthalmoplegia with a history of acute or chronic maxillary and sphenoid sinusitis. Total 38 patients had evidence of infection in the form of fever, paranasal sinus (PNS) infections, Chronic suppurative otitis media (CSOM). Total 19 (38\%) patients had a history and evidence of dehydration. Total $8(16 \%)$ patients died during the course of treatment and $42(84 \%)$ were discharged with partial and/or total recovery. Three (6\%) patients required neurosurgical intervention in the form of decompressive craniotomy. Eight (16\%) patients died with cerebral edema with transtentorial herniation. The mean age of death in male was significantly greater than in female patients with $P<0.02$. Majority of patients succumbed had sigmoid, transverse, and/or multiple sinus involvement. Patients with multiple sinus thrombosis had greater case fatality rate. Conclusions: The current study highlights the burden of CVST in the study population with headache and vomiting, which was the most common presenting complaint. The superior sagittal sinus thrombosis was the most common and bilateral cavernous sinus thrombosis was the uncommon affection in CVST. One third of female population was affected in peripartum period. The infection and/or dehydration was the most commonly associated precipitating event for development of CVST and more than one fifth of the population had evidence of hyperhomocysteinemia. Mortality was more in patients with affection of sigmoid, transverse, and/or multiple sinus involvement in male patients and superior sagittal sinus thrombosis in female patients. The treatment of CVST has to be aggressive as morbidity and mortality is relatively minimal compared with the arterial stroke.
\end{abstract}

Key words: Hyperhomocysteinemia, peripartum, sagittal sinus thrombosis, thrombosis of the cerebral venous sinuses

\section{Introduction}

Cerebral venous sinus thrombosis (CVST) is one of

\begin{tabular}{|l|l|}
\hline \multicolumn{2}{|c|}{ Access this article online } \\
\hline Quick Response Code: & Website: \\
\hline & www.ruralneuropractice.com \\
\cline { 2 - 2 } & \\
\hline
\end{tabular}

the common causes of stroke in young people. CVST is a disease with potentially serious consequences and usually affecting young to middle-aged people. Strokes in the young account for nearly $30 \%$ of all cases of stroke in India and cerebral venous thrombosis (CVT) accounts for $10-20 \%$ of these cases. Two-thirds of them develop the same in the postpartum period. There has been a drastic reduction of infection as a causative agent for CVST in recent years. Primary or idiopathic CVST are mainly caused by hypercoagulable state commonly from puerperium or from dehydration. Puerperal CVST may

Address for correspondence:

Dr. Virendra C. Patil, Department of Medicine, Krishna Institute of Medical Sciences University Karad, Satara - 415 110, Maharashtra, India. E-mail: virendracpkimsu@rediffmail.com 
account for approximately $15 \%-20 \%$ of stroke victims of younger age. CVST can be caused by a number of prothrombotic states and disorders of clotting system such as inherited cause is Protein $C$ resistance secondary to Factor V Leiden polymorphism, Protein C and S resistance, and antithrombin III deficiency. Other vasculitis such as Systemic lupus erythematosus (SLE) and polyarteritis nodosa (PAN) are also relevant in young adults. Dehydration is still most common cause puerperal CVST in our country. The purpose of this study was to analyze the characteristics of CVT in a rural hospital. CVST is an uncommon form of stroke, usually affecting young individuals. CVST represents $0.5 \%-1 \%$ of all strokes. Multiple factors have been associated with CVST, but only some of them are reversible. ${ }^{[1,2]}$ No population studies have reported the incidence of CVST. Hyperhomocysteinemia is a risk factor for deep vein thrombosis (DVT) and stroke but has not been clearly associated with an increased risk of CVST. The objectives were to study the clinical profile of the patients with CVST and to assess the risk factors involved.

\section{Materials and Methods}

This study was conducted over a period of 1 year (Jan 2011 to Dec 2011). This was a retrospective, observational, and noninterventional study. This study was conducted in the Department of Medicine at a tertiary care teaching center. The study was approved by the ethical committee of KIMS, Karad. All the patients included in the current study have documented CVST on magnetic resonance imaging (MRI) venogram. The information of all the patients fulfilling the criteria of CVST of both genders was entered in a data entry sheet. All the patients were treated in the intensive care unit under standard guidelines and protocols. Patients with diagnosis of CVST were treated in intensive care unit. Patients with diagnosis of CVST were treated with intravenous fluids to correct dehydration, decongestive agents, anticonvulsant drugs, antibiotics, low-molecular weight heparin (LMWH) (if not contraindicated), decompressive craniotomy, methylcobalamin and folic acid supplementations, and so on. All 50 patients in whom the diagnosis of CVST was confirmed by computed tomography/MRI brain venogram were included in this study. All patients underwent basic investigations, such as hemogram, electrolytes, blood sugar levels, renal function tests, and chest radiographs. Liver function tests, coagulation studies, inflammatory markers, and homocysteine levels were done in selected patients because of financial constraints. Cerebral venous system was classified into two major groups: (1) Sagittal sinuses and cortical veins draining superficial surfaces of both cerebral hemispheres. (2) Deep system comprises lateral sinus, straight sinus, and sigmoid sinus along with draining deeper cortical veins. We excluded the trauma as a factor for development of CVST from history.

\section{Statistical analysis}

SPSS software version 11 was used to analyze the data. ${ }^{[1,3,4]}$ The mean, standard deviation, and Chi-square test was used to analyze the data and $P<0.05$ was considered as statistically significant.

\section{Result}

A total of 50 patients were included in this retrospective observational study with age more than 15 years and with diagnosis of CVST. Of the total 50 patients with CVST, 21 (42\%) were males and 29 (58\%) were females with $39 \pm 10$ years and $29 \pm 7$ years, respectively.

\section{Clinical presentation}

Forty-five (90\%) patients presented with symptoms of headache and vomiting, 13 (26\%) had seizures, $12(24 \%)$ had hemiplegia, and 19 (38\%) had fever. A total of $13(26 \%)$ patients had papilledema on fundoscopy. Headache and vomiting were the most common presenting features in patients with CVST $(P<0.002)$ and next was hemiplegia and seizures [relative risk (RR): 2.36; odds ratio (OR): 14.68] [Table 1].

\section{Causative factor for CVST}

Nine $(31 \%)$ out of 29 patients had diagnosis of CVST during peripartum period (RR: 1.29; OR: 1.42). Nineteen (38\%) patients underwent test for serum homocysteine level. Total $12(24 \%)$ patients had hyperhomocysteinemia of whom $8(27.58 \%)$ were females and $4(19.04 \%)$ were male patients (RR: 1.44; OR: 1.61). Total 39 patients had evidence of infection in the form of fever, paranasal sinus (PNS) infections, and/or chronic suppurative otitis media (CSOM). Total $19(38 \%)$ patients had a history and evidence of dehydration due to fl uid loss, poor intake due to some or other reasons [Table 2 and Graph 1].

\begin{tabular}{lccc} 
Table 1: Mean age of death of cerebral venous sinus \\
\hline $\begin{array}{l}\text { Total no. of } \\
\text { deaths }(\boldsymbol{N}=4\end{array}\left(\begin{array}{c}\text { Mean } \\
\text { age (years) }\end{array}\right.$ & $\begin{array}{c}\text { Mean age of } \\
\text { males (years) }\end{array}$ & $\begin{array}{c}\text { Mean age of } \\
\text { female (years) }\end{array}$ \\
\hline Male: $(19.04)$ & $48.87 \pm 12.6$ & $58.5 \pm 9$ & $39.25 \pm 11.5$ \\
Female: $(13.79)$ & & & \\
\hline
\end{tabular}

\begin{tabular}{|c|c|}
\hline Risk factors & Total (\%) \\
\hline Dehydration & $38(76)$ \\
\hline Infection & $39(78)$ \\
\hline Pregnancy & $9(31)(n=29)$ \\
\hline Hyperhomocysteinemia & $12(24)$ \\
\hline
\end{tabular}




\section{Type of sinus involved in CVST}

Total $23(46 \%)$ patients had sagittal sinus thrombosis (RR: 1.49; OR: 1.91), 10 (20\%) had multiple sinus thrombosis, and 16 (32\%) had sigmoid/transverse sinus thrombosis. There was $1(2 \%)$ patient who had bilateral cavernous sinus thrombosis, who presented with bilateral proptosis, conjunctival congestion, and external ophthalmoplegia with a history of acute or chronic maxillary and sphenoid sinusitis. The mean duration of stay of patients admitted with CVST was $8 \pm 3$ days [Tables 3 and 4; Figures 1 and 2].

\section{Outcome of patients with CVST}

Forty-two (84\%) patients were discharged with partial and/or total recovery from illness in the form of hemiplegia or monoplegia. Of the $3(6 \%)$ patients who underwent decompressive surgery, 2 patients improved and 1 succumbed. Eight (16\%) patients died with cerebral edema with transtentorial herniation. Case fatality rate was more with sagittal sinus thrombosis [females $n=19(34.48 \%)$; death $=4(21.05 \%)$ ] in female and multiple sinus [male $n=5(23.8 \%)$; death $3(60 \%)$ ] (RR: 0.709; OR: 0.622) involvement in male patients. Total $4(19.04 \%)$ male patients $(58.5 \pm 9 \mathrm{yrs})$ and 4 (13.79\%) female (39.25 $\pm 11.5 \mathrm{yrs})$ patients succumbed. Mean age of death in male was significantly greater than in female patients with $P<0.02$. Mortality rate was relatively more in male patients. A total of $3(6 \%)$ patients required neurosurgical intervention in the form of decompressive craniotomy. Majority of patients who succumbed had sigmoid, transverse, and/or multiple sinus involvement. Patient with multiple sinus thrombosis had greater case fatality rate followed by patients with sagittal sinus thrombosis (RR: 0.57; OR: 0.4912). [Table 5 and Graph 2].

\section{Discussion}

CVST is reported to be more common in developing countries, and has been linked to pregnancy, multiparity, dehydration, and infection. Developments in imaging, diagnostic laboratory investigations, and genetics have

Table 3: Patient's and cerebral venous sinus distribution

\begin{tabular}{|c|c|c|c|c|}
\hline $\begin{array}{l}\text { Cerebral } \\
\text { venous } \\
\text { sinus } \\
\text { involved } \\
(n=50)\end{array}$ & $\begin{array}{c}\text { Total } \\
\text { patients } \\
35.66( \pm 15) \\
\text { year }(\%)\end{array}$ & $\begin{array}{c}\text { Male }(n=21) \\
41.04( \pm 11.6) \\
\text { year }(\%)\end{array}$ & $\begin{array}{c}\text { Female } \\
(n=29) \\
33.58( \pm 9.7) \\
\text { year }(\%)\end{array}$ & $\begin{array}{l}\text { Total deaths } \\
N=8 \\
(16 \%)\end{array}$ \\
\hline $\begin{array}{l}\text { Sagittal } \\
\text { sinus }\end{array}$ & $23(46)$ & $4(19.4)$ & 19 (34.48) & $\begin{array}{l}\text { Male 0: Female } \\
4(21.05)\end{array}$ \\
\hline $\begin{array}{l}\text { Sigmoid and/ } \\
\text { or transverse } \\
\text { sinus }\end{array}$ & $16(32)$ & $11(52.38)$ & 5 (17.24) & $\begin{array}{l}\text { Male } 1 \text { (9.09): } \\
\text { Female } 0\end{array}$ \\
\hline $\begin{array}{l}\text { Cavernous } \\
\text { sinus }\end{array}$ & $1(2)$ & $1(4.76)$ & Nil & $\begin{array}{l}\text { Male 0: } \\
\text { Female } 0\end{array}$ \\
\hline $\begin{array}{l}\text { Multiple } \\
\text { sinuses }\end{array}$ & $10(20)$ & $5(23.8)$ & $5(17.24)$ & $\begin{array}{l}\text { Male } 3(60) \text { : } \\
\text { Female 0 }\end{array}$ \\
\hline
\end{tabular}

Table 4: Cortical venous sinus involvement, deaths, and duration of stay

\begin{tabular}{lcc}
\hline Sinuses & Deaths & Mean duration of stay \\
\hline Sagittal & 4 & 4.75 days \\
Sigmoid/transverse & 1 & 1 day \\
Cavernous & 0 & 0 \\
Multiple sinuses & 3 & 5.6 days \\
\hline
\end{tabular}

Table 5: Deaths and cerebral venous sinus involved

\begin{tabular}{lccc}
\hline Sinuses & $\begin{array}{c}\text { Total } \\
\text { deaths }(\%)\end{array}$ & $\begin{array}{c}\text { Male } \\
(\%)\end{array}$ & $\begin{array}{c}\text { Female } \\
(\%)\end{array}$ \\
\hline Sagittal sinus $(n=23)$ & $4(21.05)$ & $\mathrm{Nil}$ & $4(21.05)$ \\
Sigmoid/transverse sinus $(n=16)$ & $1(6.25)$ & $1(9.09)$ & $\mathrm{Nil}$ \\
Cavernous sinus $(n=1)$ & $\mathrm{Nil}$ & $\mathrm{Nil}$ & $\mathrm{Nil}$ \\
Multiple sinuses $(n=10)$ & $3(30)$ & $3(60)$ & $\mathrm{Nil}$ \\
\hline
\end{tabular}

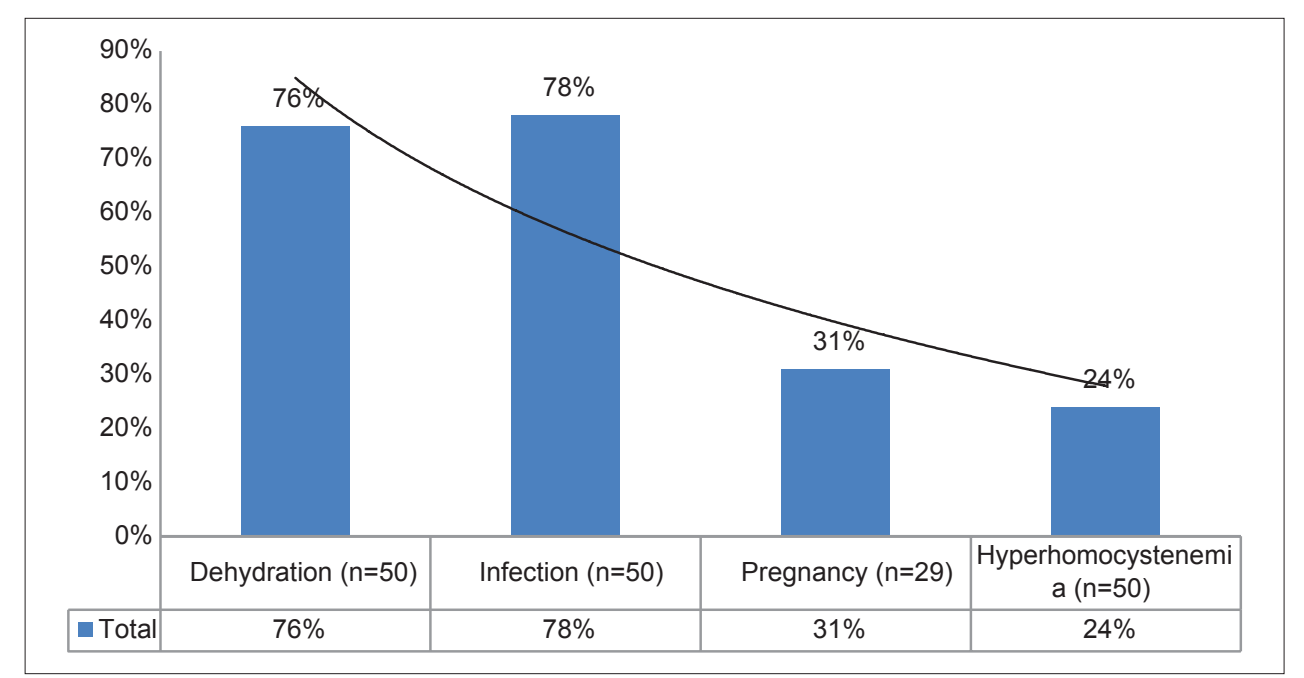

Graph 1: Risk factors for the development of cerebral venous sinus thrombosis 


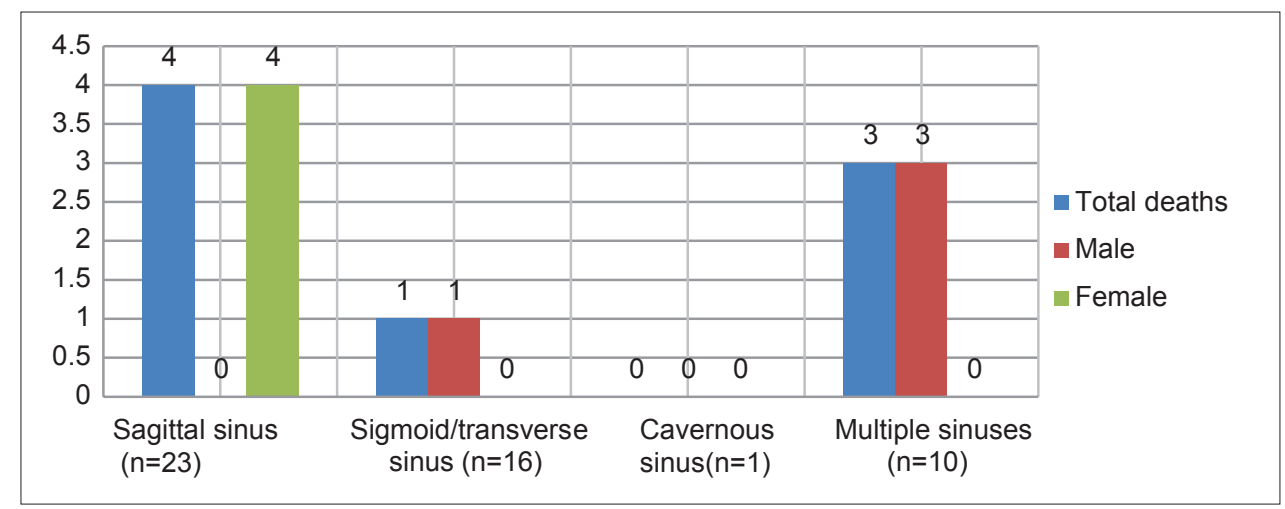

Graph 2: Type of cerebral venous sinus thrombosis and mortality

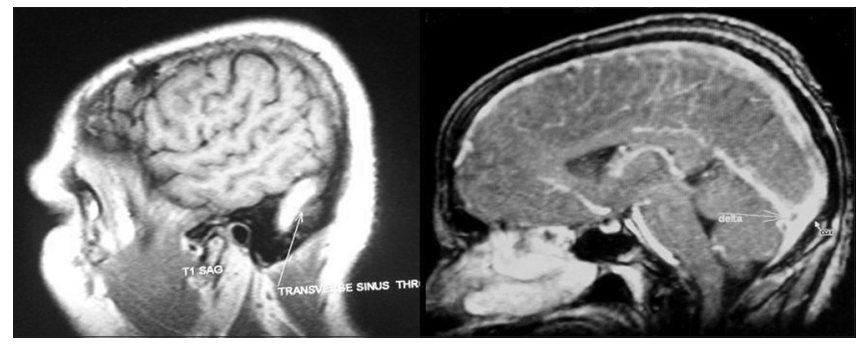

Figure 1: Magnetic resonance imaging brain venogram showing transverse sinus thrombosis and superior sagittal sinus thrombosis evidenced by empty delta sign

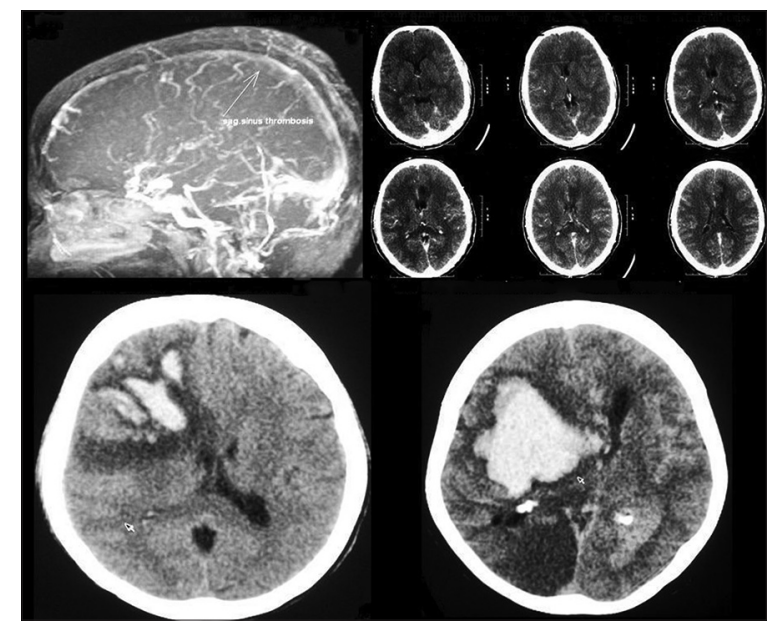

Figure 2: Magnetic resonance imaging brain venogram showing superior sagittal sinus thrombosis and hemorrhagic conversion of venous infarct and computed tomography brain showing superior sagittal sinus thrombosis evidenced by empty delta sign

provided valuable information about risk factors and clinical spectrum of CVST. We compared our experience of CVST, highlighting its diverse clinical presentations, predisposing factors, and neuroimaging with other studies from India and abroad. CVST represents $0.5 \%-1 \%$ of all strokes. According to the largest cohort study, $78 \%$ cases occurred in patients younger than 50 years. One pathological study found a prevalence of CVT of $9.3 \%$ among 182 consecutive autopsies. No population studies have reported the incidence of CVT.
Hyperhomocysteinemia is a risk factor for DVT and stroke but has not been clearly associated with an increased risk of CVT. ${ }^{[1]}$ Twenty-four percent of our population had hyperhomocysteinemia and more predominantly in female population. Lath et al. ${ }^{[5]}$ stated that the mortality in CVST, in addition to progressive thrombosis, is related to elevated intracranial pressure causing transtentorial herniation. They reported a mortality of $27 \%$. These findings are comparable with our result with a low mortality of $16 \%$. Lath et al. reported that the decompressive surgery for patients with large cerebral venous infarcts is a life-saving procedure. Similarly, in our study, $3(6 \%)$ patients underwent decompressive surgery of whom 2 patients improved and 1 succumbed. Pfefferkorn et al..$^{[4]}$ studied 32 patients with CVST with headache $(81 \%)$ being common presenting symptom. Out of 32 patients, 9 (28\%) had deep cerebral venous system thrombosis (DCVST) and $23(72 \%)$ had non-isolated DCVST. Similarly, in our study 45 (90\%) patients presented with symptoms of headache and vomiting with deep venous system thrombosis in $16(32 \%)$ (sigmoid and/or transverse sinus) and $23(46 \%)$ patients had superficial system (superior sagittal sinus) thrombosis. Azin et al.$^{[6]}$ studied 61 patients with CVST where male-to-female ratio was $1 / 3.1$. The mean age of patients was $35.6 \pm 12.1$ years. Headache was seen in $91.8 \%$ of the patients. The most frequent risk factor was oral contraceptive consumption $(62.2 \%)$. Involvement of superior sagittal sinus and lateral sinus was $80.3 \%$ and $41 \%$, respectively. The fatality rate was $14.7 \%$. Similarly, headache in $45(90 \%)$ patients was the most common presenting feature in our study. Infection dehydration hyperhomocystemia and peripartum state were the important precipitating or risk factors, either individually or in combination for development of CVST in our study with mortality of $16 \%$. In our study, 23 (46\%) (RR: 1.49; OR: 1.91) patients had sagittal sinus, $16(32 \%)$ had sigmoid and/or transverse sinus, and $10(20 \%)$ had multiple sinuses involvement. Prakash et al. ${ }^{[2]}$ reported that the most common involvement in CVST was superior sagittal 
sinus $(72 \%)$, similarly in our study $46 \%$ patients had superior sagittal sinus thrombosis. Khealani et al. ${ }^{[7]}$ stated that the obstetric CSVT has a different course and carries favorable prognosis as compared with CVT unrelated to pregnancy. There is evidence that mortality can be altered favorably by anticoagulation. Favorable outcome has been reported from $60 \%$ to $76 \%$ of cases. Favorable outcome in obstetric CVT has been attributed to the assumption that the occlusion is limited and transient with rapid recanalization or by development of collaterals. Similarly, in our study all 9 female patients in peripartum period were recovered with no mortality. Nagaraja et al. ${ }^{[8]}$ reported that strokes in the young account for nearly $30 \%$ of all cases of stroke in India and CVT accounts for 10\%$20 \%$ of these cases. Two-thirds of them develop the same in the postpartum period. Similarly, in our study about one third of female population had CVST in peripartum period. Nagarajan et al. ${ }^{[9]}$ studied total 25 patients with CVST with mean age 35.7 years with a male-to-female ratio of $13: 12$. Headache $(84 \%)$ and convulsions (47\%) were the most common clinical features. Focal neurological deficits were observed in $60 \%$ of the patients and hemiparesis was the common deficit (28\%). Infection was present in $28 \%$ cases. Superior sagittal sinus was the most frequent sinus involved. Similarly, in our study of the 50 patients with CVST, 21 (42\%) were males and 29 (58\%) were females with $39 \pm 10$ years and $29 \pm 7$ years, respectively, and $90 \%$ patients presented with symptoms of headache and vomiting with $24 \%$ having hemiplegia. Total 23 (46\%) of the patients had superior sagittal sinus thrombosis, 39 (78\%) patients had evidence of infection, and $38(76 \%)$ patients had dehydration. Similar findings were reported by Appenzeller et al. ${ }^{[10]}$ in their study of 24 patients ( 18 women, 6 men) with mean age of 29.5 years with headache (75\%) and vomiting (33\%); pregnancy or puerperium in six $(25 \%)$, and inherited thrombotic risk factors in four (17\%) patients. Pai et al. ${ }^{[11]}$ studied 612 (354 men, 219 women, and 39 children) patients with CVT with papilledema $(62 \%)$, headache $(62 \%)$, hemiparesis $(48 \%)$, seizures (31\%), and cranial nerve palsy $(7 \%)$. Superior sagittal sinus thrombosis was the most common site (74\%) associated with dehydration, sepsis, pregnancy, and puerperium, Death due to CVT was $13 \%$. These findings are comparable with our study. Haghighi et al. ${ }^{[12]}$ studied 465 patients in the age group 29.5-43.8 years with ratio of female to male being 2.79 . Headache (80\%-97\%), sensory/motor deficits (39\%$64 \%$ ), and seizure $(20 \%-62 \%)$ were the most common clinical presentations with mortality rate of $11.4 \%$. Similarly, our study had a mortality rate of $16 \%$. Mehndiratta et al.$^{[13]}$ reported that headache (80\%) is the most frequent symptom in patients with CVT with $75 \%$ being female population. The mean age among females was 27.75 years and among males was 41.5 years. Of the
4 females, 2 were postpartum. Of 2 males, 1 had hyperhomocysteinemia and one had hyperlipidemia. Similarly in our study approximately one fifth of the population had hyperhomocysteinemia predominantly in female population and about one third of the population had CVST in peripartum period. Nagaraja et al. ${ }^{[3]}$ (2007) stated that the pregnancy and puerperium increase the risk of thrombotic events, and these risks are likely to be increased in women who are carriers of thrombophilic gene polymorphisms. Prothrombin G20210A variant is reported to be the second most frequent prothrombotic polymorphism in Whites. Kalita et al..$^{[14]}$ reported that the CVST more often occurs during pregnancy, multiparity, and infection. In their study of 33 patients with CVST, mean age 37.5 years (range 16-76); 23 were female with superior sagittal sinus in 23 , the lateral sinus in 19 , straight sinus in 3 , inferior sagittal sinus in 1 , and deep venous system in 1 . Seventeen patients had multiple sinus involvements. Predisposing factors could be identified in 16 patients, and included pregnancy and puerperium in 6 , infection in 6, oral contraceptive in 2 , and dehydration in 1 each. Most CVST studies from India are of puerperal. These findings are comparable with our results. Koopman et al. ${ }^{[15]}$ stated that CVST was more frequently associated with oral contraceptive use, pregnancy, or puerperium. CVT was relatively more common in women and hormonal factors may predispose to CVT. Coutinho et al. ${ }^{[16]}$ in their study of 624 patients, $75 \%$ were women with significantly younger, with better outcome compared with male patients with CVST. These findings are comparable with our study in which death was relatively more in male than female (OR: 0.68; CI: 0.149: 3.099). Martinelli et al. ${ }^{[17]}$ in their case-control study found that the hyperhomocysteinemia is associated with a 4 -fold increased risk of CVT. These findings are comparable with our study in which $24 \%$ patients found to have hyperhomocysteinemia predominantly in female population (female-to-male ratio, 2:1). Ahmad et al. ${ }^{[18]}$ stated that inherited thrombophilias with concomitant acquired risk factors such as surgery, trauma, prolonged immobilization, pregnancy and puerperium, oral contraceptives, antiphospholipid antibodies, and hyperhomocysteinemia increase the risk of CSVT. Aaron et al. ${ }^{[19]}$ in their study of 41 patients reported that $34 \%$ had hyperhomocysteinemia with a $17 \%$ mortality. Similarly in our study, $24 \%$ of the population had hyperhomocysteinemia with an overall mortality rate of $16 \%$. Shen et al. ${ }^{[20]}$ reported a case of familial hyperhomocysteinemia-related CVST and pulmonary embolism in a 21-year-old man with elevated serum homocysteine level, that is, $46.23 \mu \mathrm{M} / \mathrm{L}$. They concluded that the cause of CVT and pulmonary embolism in this young patient was most likely related to familial hyperhomocysteinemia, precipitated by a preceding 
systemic infection. Pillai et al. ${ }^{[21]}$ in their study with CVST state that the most common risk factor was use of oral contraceptive pills (38.4\%), peurperium (23\%) and one case was of recent surgery. Eleven out of 15 patients tested for homocysteine levels had increased levels, of whom 14 were males. There were 4 deaths, out of which 2 were males. These findings are comparable with our study. Akhtar et al. ${ }^{[22]}$ stated that hyperhomocysteinemia is responsible for both arterial and venous thrombosis and it should be considered as an important risk factor for atherosclerotic vascular and venous thromboembolic diseases. Nagaraja et al. ${ }^{[3]}$ stated thathyperhomocysteinemia is a known risk factor for venous thrombosis. Hyperhomocysteinemia is associated with an increased risk of puerperal CVST occurring in Indian women and low folate levels contribute significantly to hyperhomocysteinemia. Nutritional folate and vitamin $\mathrm{B}_{12}$ deficiency can cause hyperhomocysteinemia and pregnancy may contribute to this deficiency. Rajput et al..$^{[23]}$ in their case report of CVST found that acquired hyperhomocysteinemia, presumably due to nutritional deficiencies, and was treated with $\mathrm{LMWH}$, followed by warfarin, vitamin B12, vitamin B6, and folic acid, and recovered successfully. Fernández-Moreno et al., ${ }^{[2]}$ in their case report, stated that persistent high homocysteine levels was found in patients with CVST. Seventy percent of the patients with thrombosis of the cerebral venous sinuses present hypercoagulable states, including moderate hyperhomocysteinemia. These findings are comparable with our results. Cantu et al. ${ }^{[25]}$ in their study of 45 patients found that there was a higher frequency of MTHFR mutation in patients with CVST (22\%) with high plasma concentrations of homocysteine, and low plasma folate levels were associated with an increased risk of CVST. Similarly in our study $24 \%$ patients had hyperhomocysteinemia $(23.5 \pm 6.3 \mu \mathrm{mol} / \mathrm{L})$. Hassan et al. ${ }^{[26]}$ reported that methylcobalamin, pyridoxine, and folic acid supplementation resulted in fall in homocysteine to 16.50 $\mu \mathrm{mol} / \mathrm{L}(5.46-16.2 \mu \mathrm{mol} / \mathrm{L})$. The present study highlighted the clinical profile, causative/risk factors, and outcome of patients with CVST.

\section{Conclusion}

We concluded that the treatment of CVST has to be aggressive as morbidity and mortality is relatively minimal. The present study revealed significant number of patients affected by CVST in $2^{\text {nd }}$ and $3^{\text {rd }}$ decade of life, predominantly affecting female population, approximately one third. Most common sinus affected in male was sigmoid and transverse sinus thrombosis. Sagittal sinus was most commonly affected in female population. Affections of sigmoid, transverse, and multiple sinus thrombosis had more mortality and morbidity with longer duration of stay and residual significant neurodeficit. Headache and vomiting were the most common presenting symptoms and next was seizure. Significant proportion of individual with CVST had hyperhomocysteinemia. Acute gastro enteritis, fluid loss, and presence of infection were the precipitating factors for development of CVST. Infection and dehydration are the common precipitating factors for development of CVST in developing countries, including India. The difference in the incidence is probably due to the differences in genetic makeup of different ethnic groups and race as well. Prevention and proper management of infection and dehydration with supplementation of folic acid methylcobalamin, and pyridoxine are mandatory for prevention and management of CVST along with LMWH, anticonvulsant, and decongestive drugs. Peripartum period is vulnerable for development of CVST due to the presence of infection and dehydration. Significant number of patients had CVST in their peripartum period. The meticulous intensive care management is mandatory for better outcome in patients with CVST. Methylcobalamine, folic acid, and pyridoxine supplements to the patient with hyperhomocystenemia will be helpful in the prevention of CVST. CVT is an underdiagnosed condition for acute or slowly progressive neurological deficit. Newer imaging procedures have led to easier recognition of venous sinus thrombosis, offering the opportunity for early therapeutic measures. CVST is less frequent than arterial thrombosis.

\section{Limitations of study}

Because of resource limitations, the evaluation of genetic prothrombotic factors and uncommon hematological conditions were not possible to evaluate.

\section{References}

1. Saposnik G, Barinagarrementeria F, Brown RD Jr, Bushnell CD, Cucchiara B, Cushman M, et al;; American Heart Association Stroke Council and the Council on Epidemiology and Prevention. Diagnosis and management of cerebral venous thrombosis: A statement for healthcare professionals from the American Heart Association/American Stroke Association. Stroke 2011;42:1158-92.

2. Prakash C, Bansal BC. Cerebral venous thrombosis. J Indian Acad Clin Med 2000;5:55-61.

3. Nagaraja D, Kruthika-Vinod TP, Christopher R. The prothrombin gene G20210A variant and puerperal cerebral venous and sinus thrombosis in South Indian women. J Clin Neurosci 2007;14:635-8.

4. Pfefferkorn T, Crassard I, Linn J, Dichgans M, Boukobza M, Bousser MG. Clinical features, course and outcome in deep cerebral venous system thrombosis: An analysis of 32 cases. J Neurol 2009;256:1839-45.

5. Lath R, Kumar S, Reddy R, Boola GR, Ray A, Prabhakar S, et al. Decompressive surgery for severe cerebral venous sinus thrombosis. Neurol India 2010;58:392-7. 
6. Azin H, Ashjazadeh N. Cerebral venous sinus thrombosis-Clinical features, predisposing and prognostic factors. Acta Neurol Taiwan 2008;17:82-7.

7. Khealani BA, Mapari UU, Sikandar R. Obstetric cerebral venous thrombosis. J Pak Med Assoc 2006;56:490-3.

8. Nagaraja D, Sarma GR. Treatment of cerebral sinus/venous thrombosis. Neurol India 2002;50:114-6.

9. Nagarajan E, Shankar V. Characteristics of cerebral venous thrombosis in a South Indian Rural Hospital. Int J Med Health Sci 2013;2:298-304.

10. Appenzeller S, Zeller CB, Annichino-Bizzachi JM, Costallat LT, Deus-Silva L, Voetsch B, et al. Cerebral venous thrombosis: Influence of risk factors and imaging findings on prognosis. Clin Neurol Neurosurg 2005; 107:371-8.

11. Pai N, Ghosh K, Shetty S. Hereditary thrombophilia in cerebral venous thrombosis: A study from India. Blood Coagul Fibrinolysis 2013;24:540-3.

12. Borhani Haghighi A, Ashjazadeh A, Safari A, Cruz-Flores S. Cerebral venous sinus thrombosis in Iran: Cumulative data, shortcomings and future directions. Iran Red Crescent Med J 2012;14:805-10.

13. Mehndiratta MM, Garg S, Gurnani M. Cerebral venous thrombosis--clinical presentations. J Pak Med Assoc 2006;56:513-6.

14. Kalita J, Bansal V, Misra UK, Phadke RV. Cerebral venous sinus thrombosis in a tertiary care setting in India. QJM 2006;99:491-2.

15. Koopman K, Uyttenboogaart M, Vroomen PC, van der Meer J, De Keyser J, Luijckx GJ. Risk factors for cerebral venous thrombosis and deep venous thrombosis in patients aged between 15 and 50 years. Thromb Haemost 2009;102:620-2.

16. Coutinho JM, Ferro JM, Canhão P, Barinagarrementeria F, Cantú C, Bousser MG, et al. Cerebral venous and sinus thrombosis in women. Stroke 2009; 40:2356-61.

17. Martinelli I, Battaglioli T, Pedotti P, Cattaneo M, Mannucci PM. Hyperhomocysteinemia in cerebral vein thrombosis. Blood 2003;15:1363-6.

18. Ahmad A. Genetics of cerebral venous thrombosis. J Pak Med Assoc 2006;56:488-90.
19. Aaron S, Alexander M, Maya T, Mathew V, Goel M, Nair SC, et al. Underlying prothrombotic states in pregnancy associated cerebral venous thrombosis. Neurol India 2010;58:555-9.

20. Shen HC, Lo YK, Li JY, Lai PH. Familial hyperhomocysteinemia-related cerebral venous sinus thrombosis and pulmonary embolism: A case report. Acta Neurol Taiwan 2007;16:98-101.

21. Pillai LV, Ambike DP, Nirhale S, Husainy SM, Pataskar S. Cerebral venous thrombosis: An experience with anticoagulation with low molecular weight heparin. Indian J Crit Care Med 2005;9:14-8.

22. Akhtar N, Deleu D, Kamran S. Haematologic disorders and cerebral venous thrombosis. J Pak Med Assoc 2006;56:498-501.

23. Rajput R, Dhuan J, Agarwal S, Gahlaut PS. Central venous sinus thrombosis in a young woman taking norethindrone acetate for dysfunctional uterine bleeding: Case report and review of literature. J Obstet Gynaecol Can 2008;30:680-3.

24. Fernández-Moreno MC, Castilla-Guerra L, Castella-Murillo A, Cueli-Rincón B, Fernández-Bolaños R, Gutiérrez-Tous R, et al. Hyperhomocysteinemia-related cerebral venous thrombosis. Rev Neurol 2003;37:1040-3.

25. Cantu C, Alonso E, Jara A, Martínez L, Ríos C, Fernández Mde L, et al. Hyperhomocysteinemia, low folate and vitamin B12 concentrations, and methylene tetrahydrofolate reductase mutation in cerebral venous thrombosis. Stroke 2004;35:1790-4.

26. Hassan KM, Kumar D. Reversible diencephalic dysfunction as presentation of deep cerebral venous thrombosis due to hyperhomocysteinemia and protein S deficiency: Documentation of a case. J Neurosci Rural Pract 2013;4:193-6.

How to cite this article: Patil VC, Choraria K, Desai N, Agrawal S. Clinical profile and outcome of cerebral venous sinus thrombosis at tertiary care center. J Neurosci Rural Pract 2014;5:218-24.

Source of Support: Nil. Conflict of Interest: None declared.

Announcement

\section{iPhone App}

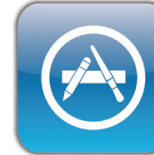

Download

iPhone, iPad application
A free application to browse and search the journal's content is now available for iPhone/iPad. The application provides "Table of Contents" of the latest issues, which are stored on the device for future offline browsing. Internet connection is required to access the back issues and search facility. The application is Compatible with iPhone, iPod touch, and iPad and Requires iOS 3.1 or later. The application can be downloaded from http://itunes.apple.com/us/app/medknow-journals/ id458064375? ls $=1 \& \mathrm{mt}=8$. For suggestions and comments do write back to us. 